\title{
Artificial Intelligence Based Efficient Smart Learning Framework for Education Platform Wei Cao ${ }^{*[1]}$, Qinan Wang ${ }^{[2]}$, Asma Sbeih ${ }^{[3]}$, FHA. Shibly ${ }^{[4]}$
}

\author{
${ }^{[1,2]}$ School of Geography Information System and Tourism ,Chuzhou University , Chuzhou \\ 239000, China \\ ${ }^{[3]}$ Palestine Ahliyauniversity, Dr computational neuroscience, engineering and information \\ department, Palestine, Bethlehem, israel \\ ${ }^{[4]}$ Senior Lecturer in Information Technology, South Eastern University of Sri Lanka.
}

*Correspondings : fyxwr111@,sina.com, 1cy@,chzu.edu.cn

\begin{abstract}
A smart learning environment is equipped with personal digital devices, wireless communication, learning platforms, and sensors that associate to provide input into Artificial intelligence systems. Artificial intelligence makes decisions about regulating the physical aspects of the environment or learning systems. These requirements may be identified by analyzing learning performance, behaviors, and the real-world and online settings in which students are situated. There are several challenges in implementing smart learning environments that are highly cost-effective, connectivity issues (Internet), impairing students' problem-solving capacity, technical challenges, e.g., malfunctioning of electronic gadgets. Hence, in this paper, Artificial Intelligence based Efficient Smart Learning Framework (AI-ESLF) has been proposed to overcome the challenges faced by a smart learning environment. This study aims to designate the smart learning environment's current concept based on AI application and examine its fundamental criteria and demonstrate how tests can be performed in this smart learning environment by case studies. The experimental results show that the suggested system enhances the prediction ratio in terms of students' learning behavior compared to other existing approaches.
\end{abstract}

Keywords: Smart Learning Environments, Artificial Intelligence, Case Study.

\section{Introduction}

Smart learning purposes at providing complete learning to learners utilizing new technology to fully prepare them for a fast-moving world where flexibility is vital [1]. Smart learning environments defined as technology-supported learning that create variations and deliver proper support (for example, feedback, guidance, tools or hints) in the right place at the right period based on distinct students' desires, which should be identified through examining their performance, behavior learning and the real-world and online settings in which they are located [2]. Smart learning environments with artificial intelligence include technology-enhanced learning, intelligent tutoring systems (ITSs), web-based learning, adaptive learning systems, context-aware ubiquitous learning, mobile learning using sensing technologies [3].Artificial intelligence may expand, strengthen, and revolutionize the way conventional classrooms provide education [4]. The incorporation of AI technology in the educational process helps educators to streamline their classroom management while at the same time supplying students with personalized guidance that fits their strengths and weaknesses [5]. Artificial Intelligence is an emerging concept associated with designing intelligent machines that can carry out functions, such as visual awareness, language comprehension, and choice of language translation, which are usually human intelligence [6]. The integration of artificial intelligence technology in the classroom world provides several advantages, such as open, collaborative, and long-term learning [7].AI may support the ability to provide personal and personalized learning to the education field [8]. Personalized learning applies to a wide spectrum of instructional services, 
learning environments, curriculum methods, and support mechanisms that meet the particular students' specific academic goals, desires, expectations, or cultural contexts [9], [10].

A smart learning system may provide learners with direct and proactive assistance from various perspectives by assessing the needs of particular learners (for instance, learning results, learning behaviors, personal, profiles variables, etc.) along with their location on the Internet or in the real world [11], [12]. Besides, it can provide learners with different personalization support, including supervision on learning, input, and resources based on their needs [13-16].It allows students to access digital resources and communicate anywhere and at any given time with learning systems. It delivers them actively with the required tools, learning guidance, tips, or suggestions at the right location, at the right period, and in the right form [18-22]. The smart learning framework may be viewed as a digitally advanced learning method that gives learners exposure to interactive services in the real world [23-25].

In this paper, the Artificial Intelligence-based Efficient Smart Learning Framework (AI-ESLF) has been proposed to overcome the challenges faced by a smart learning environment. This article gives insights into the building blocks of the AI used to progress these new education systems. The history of AI usage in education is traced. It explores the benefits of learning experiences powered by AI. Finally, features are provided for professional AI-based learning systems. The implementation of Smart learning environments (SLE) goes beyond the use of intelligent technologies. SLE encourages students to access multimedia platforms and interacts with learning templates in any area, and provides the necessary learning tips, advice, recommendations, and enabling materials actively in the right place, at the right time, and in the right form.

The main contributions of the paper are

- To propose an Artificial Intelligence-based Efficient Smart Learning Framework (AI-ESLF), overcome the challenges faced by a smart learning environment.

- Designing the research framework and case studies for smart learning to determine the course progress and learning outcomes.

- The numerical results have been performed, and the suggested system improves the prediction ratio in terms of students' learning behavior compared to other existing approaches.

The rest of the paper organized as follows, section 1 and section 2 discussed the AI application's introduction and existing approaches in creating smart learning environments. In section 3, the Artificial Intelligence-based Efficient Smart Learning Framework (AI-ESLF) has been suggested. In section 4, numerical results have been performed. Finally, section 5 concludes the research paper.

\section{Related Works}

Hongchao Peng et al. [25] introduced Personalized Adaptive Learning (PAL) for the smart learning environment. The core concept refers to a technical and effective teaching pedagogy that adapts teaching strategies in time to the changes and differences in individual performance, individual characteristics, and personal growth of learners based on real-time monitoring (enabling smart technology). A personalized adaptive learning system is developed on this basis. In addition, we tested a model of the customized learning route for the recommendation.

Mohamed Amine Chatti et al. [26] suggested the Personalization and Learning Analytics (PERLA) framework for achieving a smart learning environment. This article explores a PERLA paradigm that reflects the integration of personalization and research and provides a theoretical basis for the successful analysis of enhanced individualized learning. The key goal of the PERLA method is the systematic creation and implementation of effective metrics for personalized learning. The PERLA Framework anchors key needs that impact personalized learning while providing an extensive framework that systematically provides Learning analytics researchers and developers the means to design and create metrics to support personalized learning.

Thomas et al. [27] proposed the Partial Least Square and Structural Equation Modeling (PLS-SEM) for assessing psychological factors affecting the smart learning environment. This work shows that social support and career psychological influences have major positive effects on perceived learning in those settings. Interestingly, perceived ease of use does not affect perceived education significantly. This study explains important simulation learnings within the smart learning environment and their connexion to positive results in perceived student learning.

Nicola Capuano et al. [28] initialized Knowledge discovery and cognitive computing techniques (KD$\mathrm{CCT}$ ) for the smart learning system. Advanced features incorporated within a system include the automated classification, conceptualization, editing, versioning, incorporation, and enrichment of textual legal cases through natural language processing, creating learning paths through legal ontology, and additional functions for handling legal knowledge bases. The system has been tested on a diverse user base and successfully assessed positive 
aspects of research, including reliability, efficiency, and usability, thereby making it an effective cognitive learning tool for potential legal professionals and knowledgeable citizens.

To tackle these problems, AI-ESLF, focused on artificial intelligence, has been proposed to answer the issues facing an intelligent learning environment. This analysis aims to chart the latest approaches in the field of intelligent learning environments. This participation seeks to explore the significant obstacles to be addressed if intelligent learning experiences are corrupted. This section includes a brief overview of the proposed AI-ESLF system briefly.

\section{Artificial Intelligence-based Efficient Smart Learning Framework (AI- ESLF)}

In this paper, the Artificial Intelligence-based Efficient Smart Learning Framework (AI-ESLF) has been proposed to overcome the challenges faced by a smart learning environment.The main feature of the smart learning system is the perspective of the learner. It should be sufficiently qualified to assist students in the right period and the right location. Ubiquitous learning is the aim of the smart learning system. Only the availability/connectivity of students via sensors and other methods will fulfill this aspect. The smart learning environment is to make a forum where technology contributes to learning progression and enhances the learning experience. Smart learning environments allow teachers to plan and implement much more versatile learning modules than the traditional method. Besides, AI systems can act as mentors or tutors and ease teachers' burdens in certain cases. The AI mentors' purpose is to deliver additional support to students in learning, doing homework, preparing for the next classes. The application of AI in education and learning is expected to increase by $47.5 \%$. To this point, education has exclusively been based on human-to-human interaction, and the adoption of AI has been a bit slower than in other domains. However, it has proven to be a great helper for the teaching business. AI's ability to analyze huge amounts of data in real-time has made it easier to provide modern content or detailed learning parameters. It allows teachers to understand student performance better and arrange for more effective personalized learning plans.

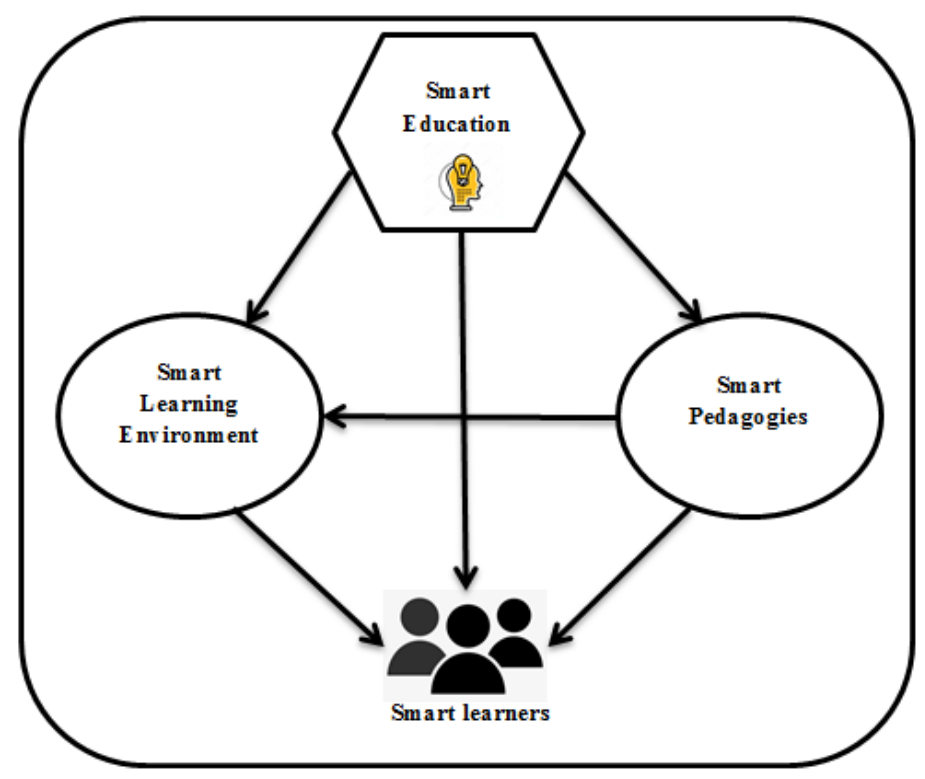

Figure 1. Smart learning environment research framework

Figure 1 demonstrates the research context for the intelligent learning environment. The model describes three main elements of intelligent learning: intelligent instruction, intelligent surroundings and intelligent learners. Intelligent education stresses the concept of good learning and is better regarded as intelligent education that tackles intelligent pedagogy as an interpersonal matter and SLE as a technological problem. Informative environments may be affected by smart pedagogics. Cognitive and metacognitive environments foster the creation of smart learners. Such capabilities are the main knowledge, skills, technological experience, and mutual intellect. There are mixed skills, talents, behaviors, and beliefs. The core concept refers to a technical and effective teaching 
pedagogy that adapts teaching strategies in time to the changes and differences in individual performance, individual characteristics, and personal growth of learners based on real-time monitoring.

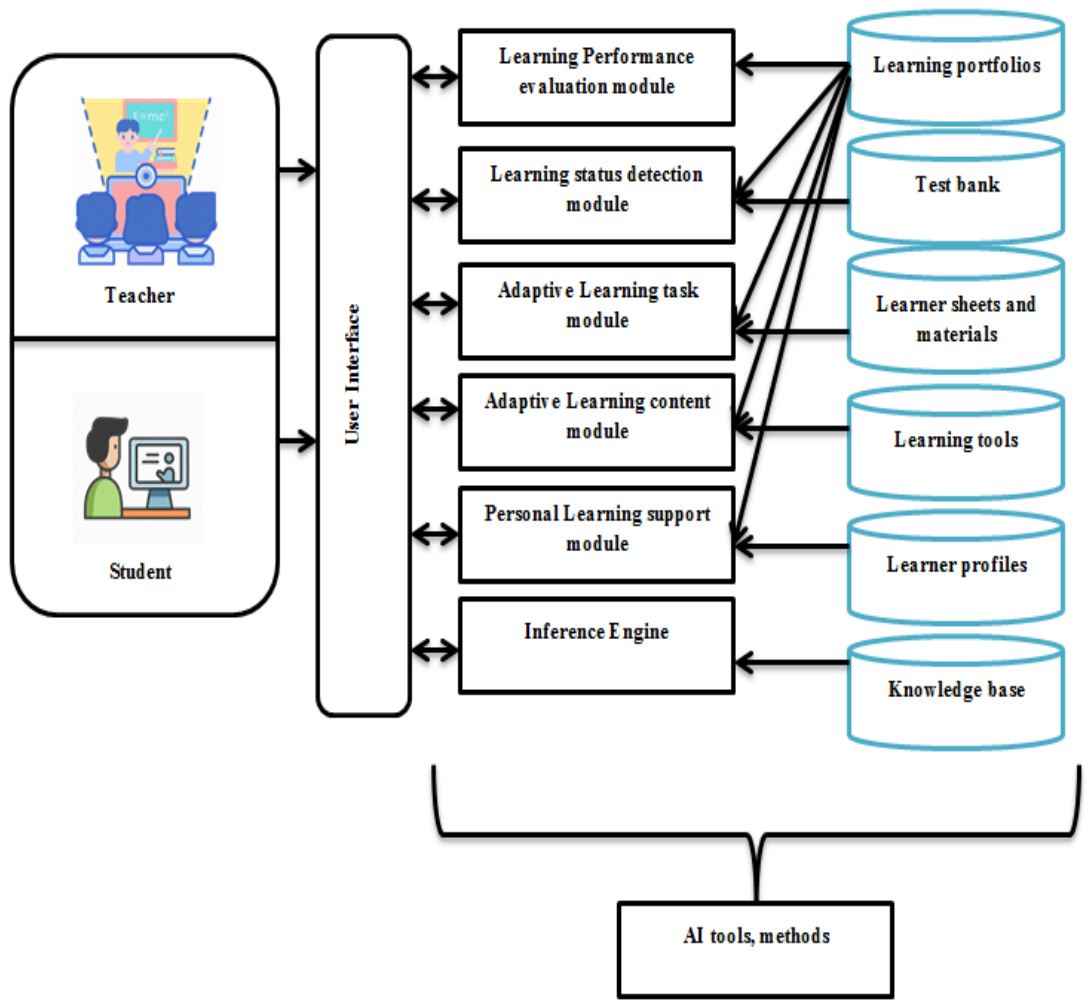

Figure 2. Application of AI in Smart Learning Environment

Figure 2 shows the application of AI in a smart learning environment. A learning status predicting module detects students' real-world status, such as learning behaviors and locations and environmental contexts, such as humidity and temperature, through linking to sensing devices-a module to measure learner activities' success and monitor the outcomes of real-world or online tests. An Adaptive learning system module assigns learners learning assignments in each discipline based on their academic and academic performance. The learning challenge may be an online study task requiring students to find knowledge on the Internet or an undertaking for real-life study, requiring them to fulfill the real goal, ask them about stuff, and find additional content on the Web the Real World.

Under this module's guidance, students perform the most feasible and relevant tasks of study often in the appropriate position at the right time by looking at their learning state and personal variables. An interactive module provides students with learning resources. The learning framework proposes and maintains learning materials based on learning development, learning results, personal variables, and the real-world status of individual learners and adapts the interface to individual learners' needs. A personal learning support program gives learners learning assistance depending on their learning requirements. The learning may guide the learning materials or task, an overview of the learning process, feedback on the job, or a Mindtool to help you understand effectively.

An information base collection maintains student accounts, portfolios, materials, assessments, and feedback. By analyzing the knowledge in the learning portfolios, the individual students learn the ideas or skills they need to acquire. Thus, some techniques and resources are needed to provide them with the most productive teaching assignments. An inference engine and knowledge foundation analyze the "values" of assignments, approaches and techniques, and their analytics variations. The know-how is a collection of the information and expertise of teachers. It could include decisions made by assessing good cases and trying cases.

The student profile identified multi-angle and multi-dimensional learning individualities. It includes parameter analysis and impacts such as data selection, user conduct, student accounts creation, data cleaning, and analysis.

The preliminary study on the learner's profile is the learners in the college or Electronic learning platform. Let's preassume the learner set as 


$$
W=\left\{w_{j}^{i} \mid w_{1}^{i}, w_{2}^{i}, \ldots w_{m}^{i}\right\}
$$

As inferred from the equation (1) where $w_{j}^{i}$ is the learner categorized by age, $j$ denotes the individual, $i$ indicates the learner's age. According to age, learner profile information is predicted and further classified into the students learning characteristics.

This paper focuses on the learner's characteristics from online learning behavior subsequent exploration to comprehend the performance of the learner. The fundamental of learning behavior is the task of online learning behavior. The student profile behavior set is stated as

$$
A=\left\{a_{j} \mid a_{1}, a_{2}, \ldots a_{m}\right\}
$$

As shown in equation (2) where $a_{j}$ denotes different behavior and includes text learning, learning goal, making notes, online practice, exchange interaction, download courseware, communicate through E-mail, the question online, and rest or listen to music.

Since online learning is the time process with online learning behavior, it is a significant variable to assess online learning quality. In particular, it reproduces the level of concentration on learning. The timeslot set in the learner profile is stated as

$$
T=\left\{t_{j} \mid t_{1}, t_{2}, \ldots t_{m}\right\}
$$

As discussed in equation (3) where $t_{j}$ denotes different duration.

The student's profile has a pattern to direct us in the study of the online learning process of students. Data acquisition involves four categories, like weblog data, learner user registration data, learning content preference data, and learning behavior information. Due to the interloping of irregular values, data misrepresentation often enhances the accuracy of data exploration and guarantees data consistency.

The student profile has a trend that directs us in the study of students' online learning. Data acquisition covers four categories: weblog data, register data for pupils, data preferences for learning material, and information on behavior. Sometimes, data misrepresentation improves the data scan's precision and ensures continuity of the data regardless of interlinkages of irregular values.

Parameter induction is an effective procedure for gathering data source pre-treatment. Supposing the actual data field to $\left\{H_{1}, H_{2}, \ldots H_{M_{H}}\right\}$, where $B$ means desirable property, and $M_{H}$ denotes the dimensions of the actual data filed. The parameter induction approach is stated as a sig using the property order of data pre-processing to stretch every the anticipated elements, cleaning the data to get the subsequent outcomes,

$$
\left\{B_{1}, B_{2}, \ldots B_{M_{R}}\right\}=\operatorname{sig}\left\{H_{1}, H_{2}, \ldots H_{\mathrm{m}}\right\}
$$

As shown in equation (4) where $M_{B}$ denotes significant property for the dimension data field. 


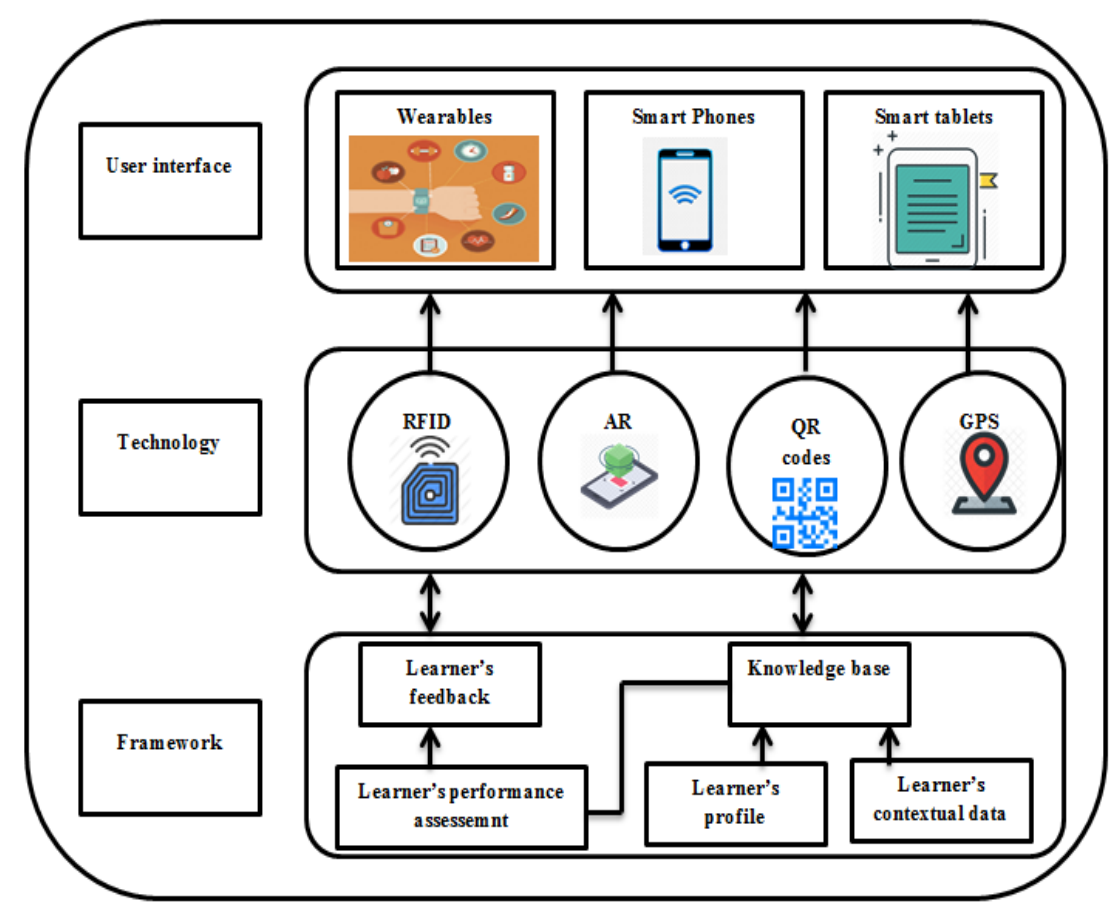

Figure 3. The architecture of the proposed AI-ESLF method

Figure 3 shows the architecture of the proposed AI-ESLF method.Connectivity is pervasive in smart devices. The embedded GPS in these devices works smoothly and allows the learner to map the learning material as needed. Smart apps may be used to customize the information base according to the student's needs. Smart apps liberate students from the disadvantage of a hardwired computer's set data point.The digital learning experience is about learners obtaining data from mobile apps. Another aspect in which teachers recognize how they think and sound through actuators, new sensors, and wearables. The learner knows how they feel and understand. Intelligent tools allow teachers to grasp and encourage students to change their minds. Augmented reality is a modern concept utilized for teaching/learning. AR utilizes the concept of 3D layering space that creates modern experiences of the world. RFID serves as a concept progression enabler. This is one of the fast-growing technologies that impact society tremendously. The RFID tag is a small antenna microchip.

Further, An interactive module provides students with learning resources. A personal learning support program gives learners learning assistance depending on their learning requirements. The learning may guide the learning materials or task, an overview of the learning process, feedback on the job, or a Mindtool to help you understand effectively.

Using electromagnetic wave tags, RFID readers relay commands and energy to active tags. Induction coupling or electromagnetic wave contact is based on the connection from one marker to the other. The scan machine (reader) transforms the reflected radio waves back from the tag into digital content, which can then be transmitted to the computer network. RFID om smart learning environment essentially allows end-user and context information to be found. The QR Codes are machine-readable contains black and white squares, which store URLs or other camera or smartphone content. QR codes may be used to boost smart learning results. QR codes may support students in numerous ways. Students can view and acquire descriptive knowledge, or they may have access to information about their physical role. In various tests, learners may use QR codes by obtaining GPS co-ordinates and using quick response website content to provide a time-consuming alternative for a contact process. The Global Positioning System is a satellite directing device. The GPS receiver signal can also control the location, speed, and times of numerous vehicles, persons, and other devices. At a certain time, the GPS satellite records the route by repetitive signals. Signals fly to a neighboring time zone at a light level since few satellites are invisible. The GPS direction can be determined by calculating how long it takes to hit the target.The similarity in the different student's behavior set has been calculated via the Jaccard coefficient similarity likened with the characteristics of online behavior and period of students, the same characteristics categorized as a class, and the variance features categorized to diverse classes. The similarity between the behavioral characteristics of various student objects fits into non-numeric objects; this paper adopts the Jaccard coefficient evaluated similarity.

$$
v_{j i l}=\operatorname{jaccard}\left(w_{j} * a_{l} * w_{i} * a_{l}\right)=\frac{\left|w_{j} \cdot a_{l} \cap w_{i} \cdot a_{l}\right|}{\left|w_{j} \cdot a_{l} U w_{i} \cdot a_{l}\right|}
$$


As derived in equation (5) where $w_{j} \times a_{l}$ denotes the behavior $a_{l}$ of students $w_{j}$ and $w_{i}$. Supposing the student $w_{j}$ belongs to the knowledge base, then compare $w_{j}$ and $w_{i}$. If $w_{j}$ and $w_{i}$ similarity variances are high, and it can be auxiliary to the knowledge base as a new class,

Students similarity is stated as,

$$
V_{j i}=\operatorname{sim}\left(w_{j}, w_{i}\right)=\frac{1}{N} \sum_{l=1}^{N} v_{j i l}
$$

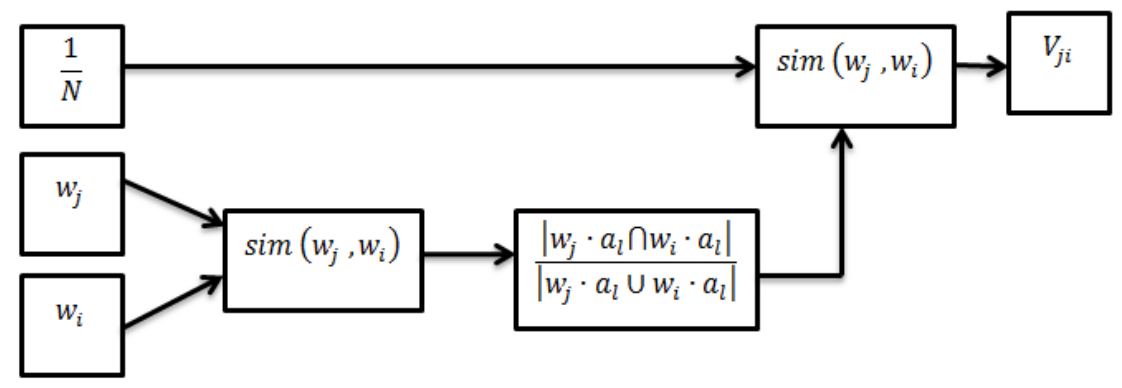

Figure 4. Students Similarity

As inferred from the equation (6) and figure 4, where $\operatorname{sim}\left(w_{j}, w_{i}\right)$ denotes similarity between students $w_{j}$, and $w_{i} ; N$ denotes the behavior dimension parameter of student set $W ; v_{j i l}$ denotes the similarity of property $l$ between students $w_{j}$ and $w_{i}$ and $j \neq i$.

According to similarity evaluation, Matrix $_{\text {sim }}$ has been obtained as follows:

$$
\text { Matrix }_{\operatorname{sim}}=\left[\begin{array}{ccccccc}
1 & V_{12} & V_{13} & & \cdots & \cdots & V_{1 M} \\
0 & 1 & V_{23} & \cdots & \cdots & \cdots & V_{2 M} \\
0 & 0 & 1 & & \cdots & \cdots & V_{3 M} \\
& \vdots & & \ddots & & \vdots & \\
& & & & 1 & \vdots & \vdots \\
& & & \cdots & 0 & \ddots & V_{M-1, M} \\
0 & & & & 0 & 0 & 1
\end{array}\right]
$$

As shown in equation (7), the triangular matrix, where $0<V_{j i}<1, V_{j i}$ indicates row $i$, and line $j$, and it is the behavior characteristics similarity between learners $j$ and $i$.

Let's define every progress having $j$ students and every student having $m$ characteristic values,

Predictive value is

$$
D=\left\{D_{1}, \ldots D_{m}\right\} \in V^{j \times m}
$$

$$
E=f(D) \in V^{j}, e \in\{0,1\}
$$

As discussed in equation (8) and (9) where $D_{m}$ indicates a course, $e=0$ means it improbable to get a certificate, and $f(D)$ are predictive functions.

When revising the lecture content for learning, students can try to answer the questions registered by the teacher. Equation 10 shows the student score in every question based on how many attempts has made until the right answer that describes students knowledge level on the subjects,

$$
L=N /(P-1) *(P-M)
$$

As shown in equation (10), where $L$ indicates the knowledge probability, $N$ denotes the maximum knowledge probability, $P$ indicates the Number of alternatives to th quiz, $M$ denotes attempt in which the student succeeded. 
The proposed Artificial Intelligence-based Efficient Smart Learning Framework (AI-ESLF) for a smart learning environment achieves high performance and predicts student online learning behavior compared to other existing methods. This paper focuses on the learner's characteristics from online learning behavior subsequent exploration to comprehend the performance of the learner. The fundamental of learning behavior is the task of online learning behavior.

\section{Numerical Results}

This paper presents the Artificial Intelligence-based Efficient Smart Learning Framework (AI-ESLF); the student's data has been collected from smart learning environments like colleges and universities. The following performance metrics have been performed using the AI-ESLF method.

\subsection{Overall Performance Ratio}

The proposed model is producing data that guides teachers and students to exploit success and reduce failure. It is rather a significant tool that supports to determine the actual abilities and boundaries of the subject's intricate in the learning progression. The utilization of the open model based on Artificial intelligence allows good performance for learners since they are concentrated on their limitations, therefore enhancing the efficiency of the knowledge acquisition procedure. For students, particular features allow the visualization of the performance level at various points in the model. The proposed AI-based smart learning model enhances the overall performance when compared to other existing approaches. Figure 5 demonstrates the overall performance of the suggested AIESLF system.

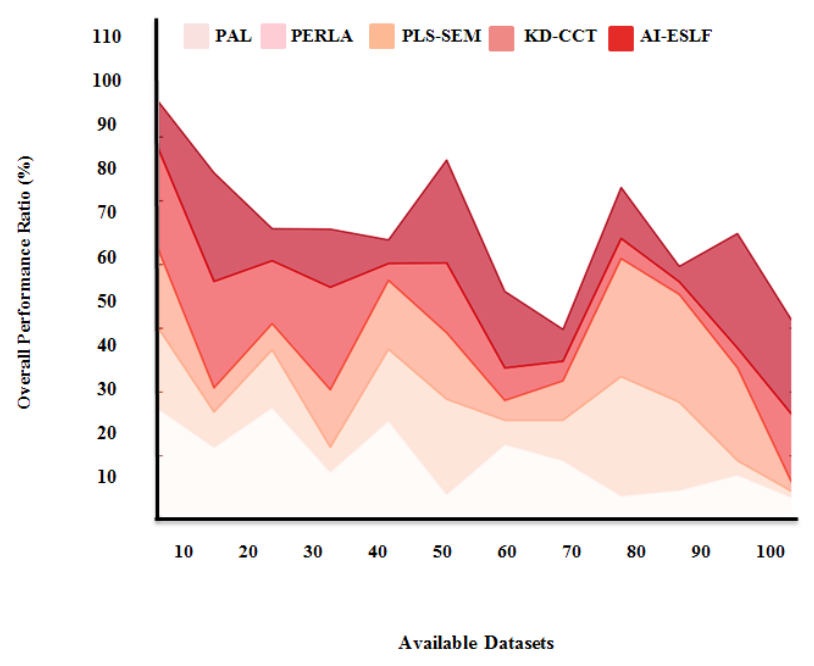

Figure 5. Overall performance Ratio

\subsection{Learning attitude analysis}

Learning behavior is a two-way relationship between students, intending to bring about improvements that are fairly consistent in the information and skills they can do. Students may choose learning goals and success, and they choose an appropriate learning strategy. In addition, students can use interactive platforms to collaborate and to learn online or face-to-face interaction in specific subjects. Learning habits can be summed up as information retrieval behavior, information processing, data sharing, and contact between people. According to intelligent (practical) abilities, learning results, oral (declaration) cognitive, knowledge technique, motor skills, and behavior. Students need to select learning goals, success in learning, and self-learning strategies associated with learning behavior in smart learning. Figure 6 shows the learning attitude analysis in a smart learning environment using the proposed AI-ESLF method. 


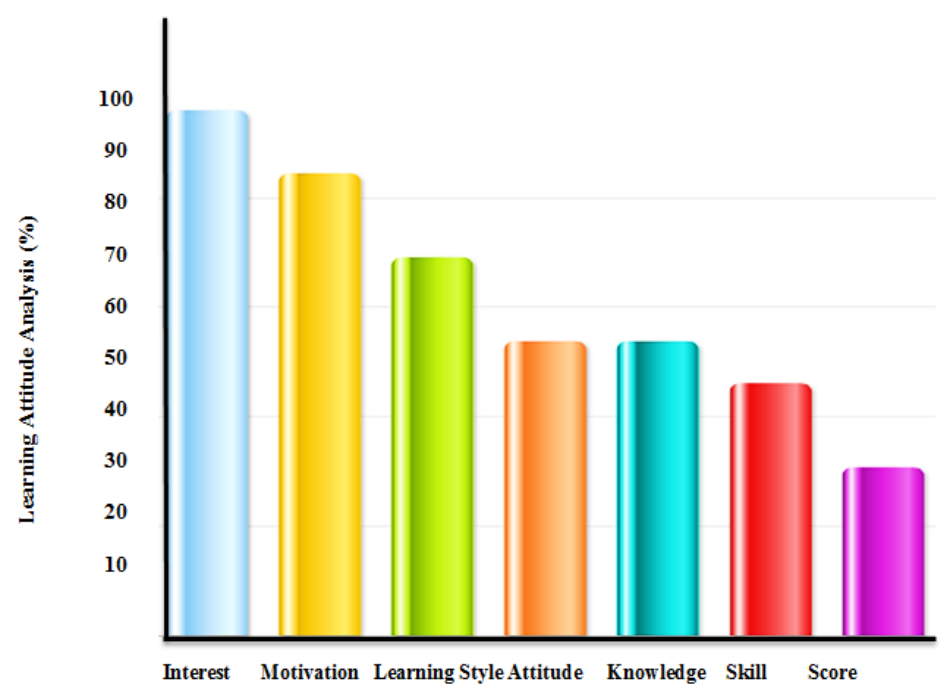

\subsection{Prediction ratio evaluation}

Figure 6. Learning attitude Analysis

Learning behavior prediction and learning pattern analysis are essential aspects of an integrated realworld environment. As the smart learning environment can record any aspect of the student's learning behaviors, it helps give a substantial opportunity to collect useful and accurate information by studying these practices. Longterm observations and study of learning behavior, most importantly, could be done. The proposed AI-ESLF method improves the prediction ratio in terms of students learning behavior in smart learning environments compared to other existing approaches.Figure 7 validates the prediction ratio using the suggested AI-ESLF model.

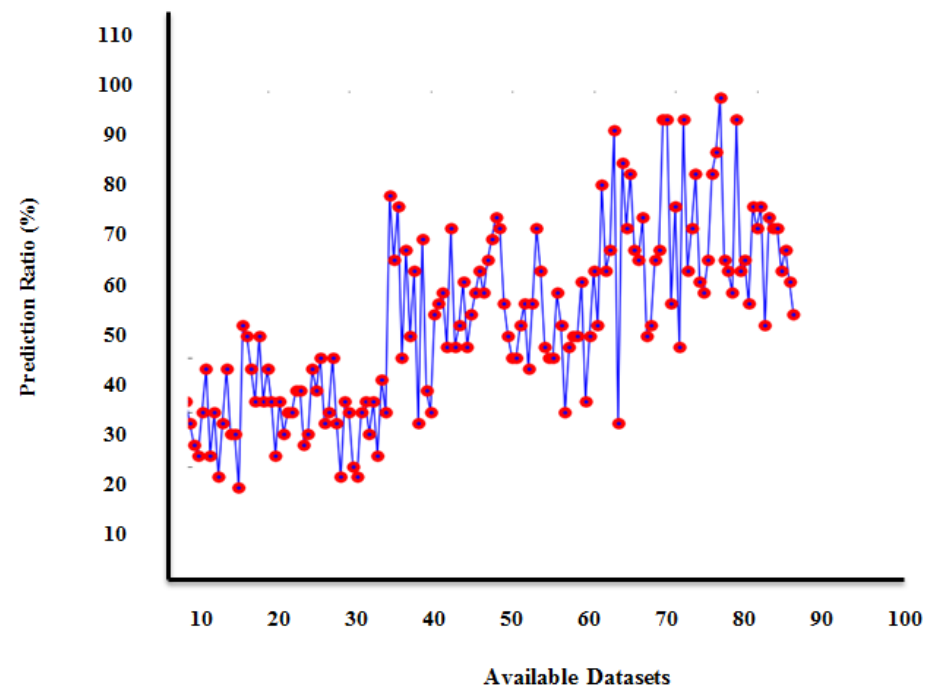

\subsection{Reliability ratio}

Figure 7. Prediction Ratio

Reflection and feedback can criticize solutions or performance and provide timely meaning and feedback to learners based on participants' progress and profile and existing learning assignments. This means AI-based smart learning if students can control their learning (self-regulated learning). The ability to regulate themselves, manage, and schedule their learning activities is known as self-regulation learning. These processes of control have been called self-regulated learning. The proposed AI-ESLF method enhances the reliability ratio when compared to other existing approaches. Figure 8 validates the reliability ratio of the suggested AI-ESLF model. 


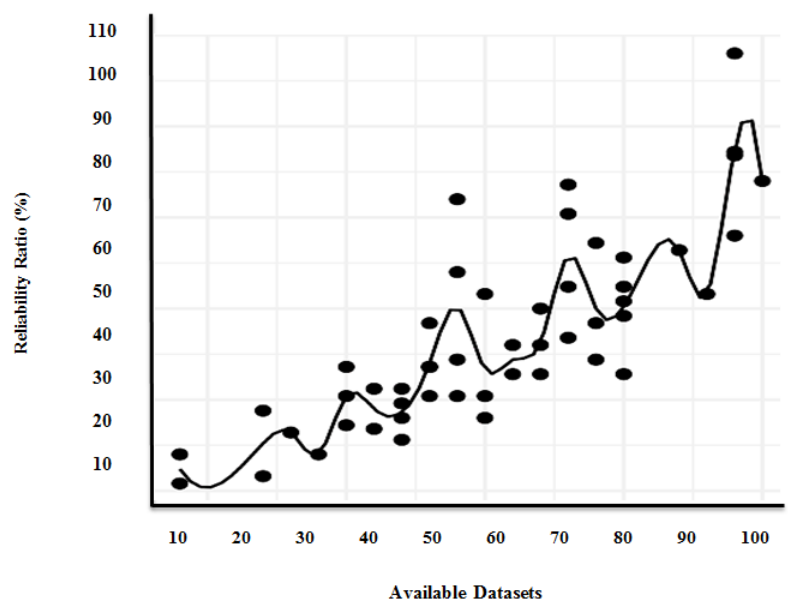

\subsection{Efficiency Ratio}

Figure 8. Reliability Ratio

A hybrid solution is created in the future of learning with AI, in which AI can assist teachers to fulfill multiple students' different needs efficiently at the same time. The role of teachers is evolving in the future. Educators may invest their time in teaching and learning more effectively and efficiently. A teacher's repetitive roles are assigned to the $\mathrm{AI}$, and human beings perform diverse and creative activities. Using AI in their smart classrooms, teachers can teach their subjects more profoundly and cultivate students' interest and creativity. The proposed AI-ESLF method enhances the efficiency ratio in smart learning environments when compared to other existing approaches. Figure 9 validates the efficiency ratio using the suggested AI-ESLF model.

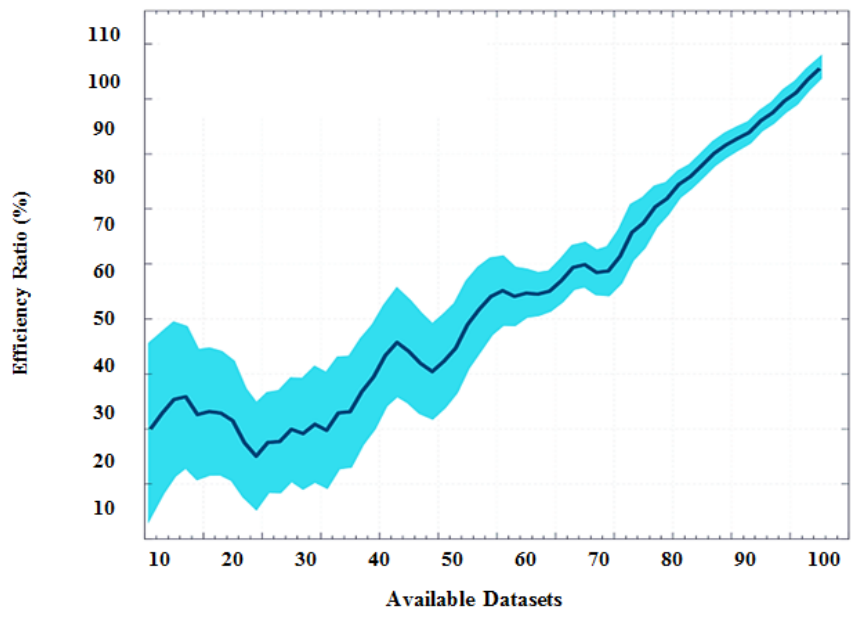

Figure 9. Efficiency ratio

The proposed Artificial Intelligence-based Efficient Smart Learning Framework (AI-ESLF) for smart learning environment achieves high performance, predicts student online learning behavior when compared to other existing Personalized Adaptive Learning (PAL), Personalization and Learning Analytics (PERLA) framework, Partial Least Square and Structural Equation Modeling (PLS-SEM), Knowledge discovery and cognitive computing techniques (KD-CCT) methods

\section{Conclusion}

This paper presents the Artificial Intelligence-based Efficient Smart Learning Framework (AI-ESLF) to overcome the challenges faced by a smart learning environment. Integratingthe Internet and wireless devices for any student in the class was essential for enhancing the learning experience. They were used through wireless resources to undertake inquiries and collaborate. For classroom students, digital monitors and screens were necessary, or they shared learning results and encouraged interaction.It focuses on contextual, personalized, and streamlined learning to facilitate the growth of student knowledge in smart environments. Smart teaching must face various obstacles, including pedagogical philosophy, the advancement of education technology and teacher 
leadership, social and cultural structures, through the growth of innovations and inside modern society. The experimental results show that the proposed AI-ESLF method enhances the prediction and performance ratio in terms of students' online learning behavior and interaction compared to other popular methods.

\section{References}

[1] Hwang GJ, Fu QK. Advancement and research trends of smart learning environments in the mobile era. International Journal of Mobile Learning and Organisation. 2020;14(1):114-29.

[2] Cebrián G, Palau R, Mogas J. The smart classroom as a means to the development of ESD methodologies. Sustainability. 2020 Jan;12(7):3010.

[3] Normadhi NB, Shuib L, Nasir HN, Bimba A, Idris N, Balakrishnan V. Identification of personal traits in adaptive learning environment: Systematic literature review. Computers \& Education. 2019 Mar 1;130:168-90.

[4] Pan HL, Wang LY, Chien MF. Developing a profession competence indicator system for teachers of elementary schools and junior high schools. Educational Research \& Information. 2004;12(4):129-68.

[5] Poniszewska-Maranda A, Kaczmarek D, Kryvinska N, Xhafa F. Studying usability of AI in the IoT systems/paradigm through embedding NN techniques into mobile smart service system. Computing. 2019 Nov;101(11):1661-85.

[6] Al_Janabi S. Smart system to create an optimal higher education environment using IDA and IOTs. International Journal of Computers and Applications. 2020 Apr 2;42(3):244-59.

[7] García-Tudela P, Prendes-Espinosa M, Solano-Fernández I. Smart Learning Environments and Ergonomics: An Approach to the State of the Question. Journal of New Approaches in Educational Research (NAER Journal). 2020 Jul 15;9(2):245-58.

[8] Hassan AE, Ibrahim ME. Designing quality e-learning environments for higher education. Educational Research. 2010 Jul;1(6):186-97.

[9] Kumar NS. Implementation of artificial intelligence in imparting education and evaluating student performance. Journal of Artificial Intelligence. 2019;1(01):1-9.

[10] Elhoseny H, Elhoseny M, Abdelrazek S, Riad AM. Evaluating learners' progress in smart learning environment. InInternational Conference on Advanced Intelligent Systems and Informatics 2017 Sep 9 (pp. $734-$ 744). Springer, Cham.

[11] Mittelstaedt E, Wiepcke C. Blended Learning to Enhance Digital and Entrepreneurial Competencies in Smart Education. Новий Колегіум. 2019(2):30-3.

[12] Abdel-Basset M, Manogaran G, Mohamed M, Rushdy E. Internet of things in smart education environment: Supportive framework in the decision-making process. Concurrency and Computation: Practice and Experience. 2019 May 25;31(10):e4515.

[13] Chen Y, Lin T, Muthu B, Sivaparthipan CB. Study on ethical dilemmas faced by teaching professionals in rural environments. Current Psychology. 2020 Jun 13:1-0.

[14] Yufeia L, Salehb S, Jiahuic H, Syed SM. Review of the Application of Artificial Intelligence in Education. integration. 2020;12(8).

[15] Lin YT. Impacts of a flipped classroom with a smart learning diagnosis system on students' learning performance, perception, and problem solving ability in a software engineering course. Computers in Human Behavior. 2019 Jun 1;95:187-96.

[16] Bandaragoda T, Adikari A, Nawaratne R, Nallaperuma D, Luhach AK, Kempitiya T, Nguyen S, Alahakoon D, De Silva D, Chilamkurti N. Artificial intelligence based commuter behaviour profiling framework using Internet of things for real-time decision-making. Neural Computing and Applications. $2020 \mathrm{Feb}$ 20:1-5.

[17] Ashar M, Rosyid H, Taufani A, Nasir K. Ubiquitous Learning Environment for Smart Improving Disruption Activities in Classroom on Media.

[18] Nieto Y, García-Díaz V, Montenegro C, Crespo RG. Supporting academic decision making at higher educational institutions using machine learning-based algorithms. Soft Computing. 2019 Jun;23(12):4145-53.

[19] Bogusevschi D, Muntean C, Muntean GM. Teaching and learning physics using 3D virtual learning environment: A case study of combined virtual reality and virtual laboratory in secondary school. Journal of Computers in Mathematics and Science Teaching. 2020 Jan;39(1):5-18.

[20] Pliakos K, Joo SH, Park JY, Cornillie F, Vens C, Van den Noortgate W. Integrating machine learning into item response theory for addressing the cold start problem in adaptive learning systems. Computers \& Education. 2019 Aug 1;137:91-103.

[21] Shorfuzzaman M, Hossain MS, Nazir A, Muhammad G, Alamri A. Harnessing the power of big data analytics in the cloud to support learning analytics in mobile learning environment. Computers in Human behavior. 2019 Mar 1;92:578-88. 
[22] Chui KT, Fung DC, Lytras MD, Lam TM. Predicting at-risk university students in a virtual learning environment via a machine learning algorithm. Computers in Human Behavior. 2020 Jun 1;107:105584.

[23] Akhrif O, Benfares C, Hmina N. Collaborative Approaches in Smart Learning Environment: A Case Study. Procedia Computer Science. 2020 Jan 1;175:710-5.

[24] Alajmi Q, Al-Sharafi MA, Abuali A. Smart learning gateways for Omani HEIs towards educational technology: benefits, challenges and solutions. Int. J. Inform. Technol. Lang. Stud. 2020;4(1):12-7.

[25] Peng H, Ma S, Spector JM. Personalized adaptive learning: an emerging pedagogical approach enabled by a smart learning environment. Smart Learning Environments. 2019 Dec;6(1):1-4.

[26] Chatti MA, Muslim A. The PERLA framework: Blending personalization and learning analytics. International Review of Research in Open and Distributed Learning. 2019;20(1).

[27] Thomas LJ, Parsons M, Whitcombe D. Assessment in Smart Learning Environments: Psychological factors affecting perceived learning. Computers in Human Behavior. 2019 Jun 1;95:197-207.

[28] Capuano N, Toti D. Experimentation of a smart learning system for law based on knowledge discovery and cognitive computing. Computers in Human Behavior. 2019 Mar 1;92:459-67. 